\title{
1,2,3-Triazoles as Amide-bond Surrogates in Peptidomimetics
}

\author{
Ibai E. Valverde ${ }^{\S}$ and Thomas L. Mindt* \\ \$SCS-Metrohm Foundation Award for best oral presentation
}

\begin{abstract}
Triazoles represent a class of heterocycles with interesting properties for application in peptide sciences since they closely resemble amide bonds while being stable to enzymatic degradation. These characteristics make 1,2,3-triazoles promising candidates as amide-bond surrogates for the development of novel peptidomimetics with potentially improved biological characteristics. Despite the potential of the heterocycle as an amide-bond isoster, only few examples of triazole-based peptidomimetics can be found in the literature. With the intention to promote this new and promising strategy for peptide modification, this review summarizes synthetic methods available for the facile preparation of $\alpha$-amino acid and $\alpha$-amino alkyne building blocks and their use for the incorporation of 1,4-disubstituted 1,2,3 triazoles into the backbone of peptides mediated by the $\mathrm{Cu}(\mathrm{I})$-catalyzed alkyne-azide cycloaddition (CuAAC). In addition, examples of the successful amide-to-triazole substitution in biologically active peptides are presented.
\end{abstract}

Keywords: Amide mimics · Click chemistry · CuAAC · Peptidomimetics · 1,2,3 Triazoles

\section{Introduction}

Peptides are a major class of pharmacologically active compounds, which benefit from high specificity and low toxicity. As such, they offer an attractive alternative to small molecule therapeutics. However, a number of peptides of medicinal interest suffer from poor metabolic stability as a result of rapid degradation by proteases in vivo. As a consequence, the discovery of new structural elements that mimic effectively the physicochemical properties of an amide bond has been a long-time challenge for chemists. The replacement of amide bonds by isosteres or bioisosteres in biologically active peptides can provide access to new peptidomimetic structures with improved biological properties. In this context, peptide backbone modifications have become a common strategy for structure-activity relationship studies of bioactive peptides and peptide-based drug design. [1] Tools available for medicinal and peptide chemists are manifold and range from amide-related functional groups such as esters, thioesters, thioamides, hydrazides, or ureas, ${ }^{[2]}$ to a variety of heterocycles including tetrazoles, oxazoles, or 1,2,4-triazoles (Fig. 1). ${ }^{[3]}$

${ }^{*}$ Correspondence: Prof. Dr. T. L. Mindt University of Basel Hospital

Department of Radiology and Nuclear Medicine

Division of Radiopharmaceutical Chemistry

Petersgraben 4

$\mathrm{CH}-4031$ Basel

Tel.: +41615565380

E-mail: Thomas.Mindt@usb.ch
Of the heterocyclic mimetics of amide bonds reported, 1,2,3-triazoles are of particular interest because they share

both electronic and topological characteristics with their natural counterpart (Fig. 2). ${ }^{[4]} 1,2,3$ Triazoles and amide bonds are

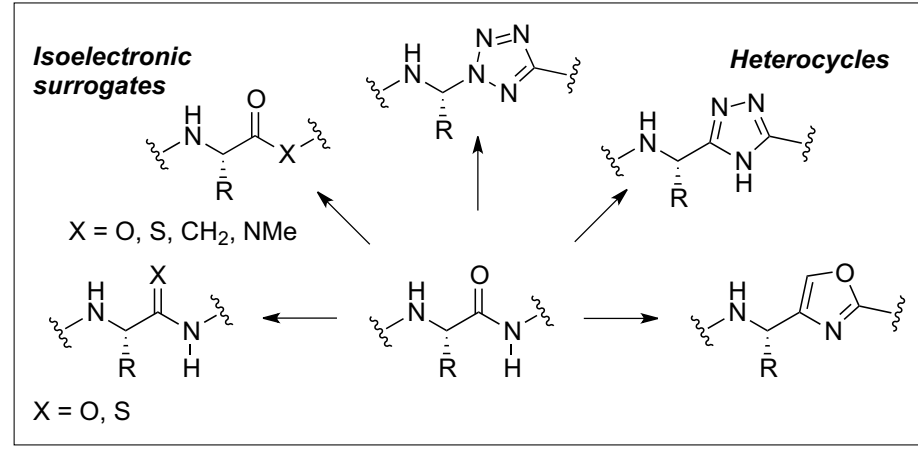

Fig. 1. Representative examples of amidebond surrogates used in drug discovery.
A

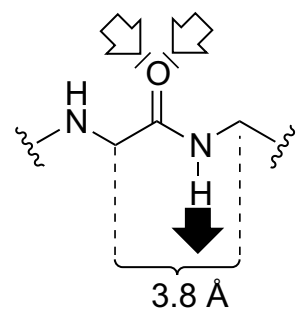

B

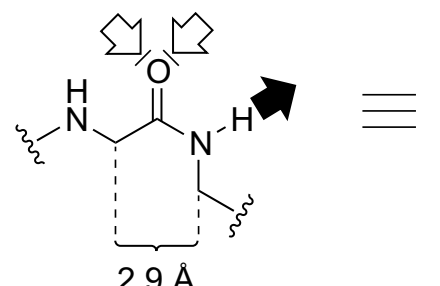

$2.9 \AA$<smiles>[Z6]NC(I)c1nnn(C2CC2)c1I</smiles>

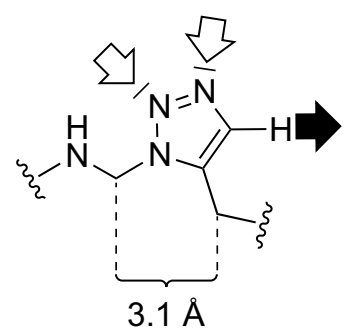

Fig. 2. Similarities of trans-amides and cisamides with 1,4- (A), and 1,5-disubstituted 1,2,3-triazoles (B). 
both planar and of similar size, possess a high dipolar moment, and feature similar $\mathrm{H}$-bonding capabilities. ${ }^{[5]}$ In contrast to amide bonds, the disubstituted heterocycle is resistant to cleavage by proteases. All these characteristics make 1,2,3-triazoles attractive amide-bond surrogates for peptide backbone engineering.

The discovery of the copper(I)-catalyzed azide-alkyne cycloaddition (CuAAC) by the groups of Meldal and Sharpless in 2002 provided an easy access to 1,4-disubstituted 1,2,3-triazoles (Scheme 1) and thus, to peptidotriazoles, a new class of backbone-modified peptidomimetics. ${ }^{[6]}$ In 2005, Fokin and co-workers reported the catalytic transformation of azides and alkynes mediated by ruthenium(II), which gives selective access to 1,5-disubstituted 1,2,3 triazoles (RuAAC) as a structural variation. ${ }^{[7]}$ Despite the major interest of the scientific community in CuAAC and RuAAC, relatively few examples of triazole-based peptide backbone modifications have been reported. A possible reason for the moderate use of 1,2,3-triazoles in peptide-based drug design could be the lack of either commercially available building blocks or, alternatively, the limited number of successful examples of amide-to-triazole substitutions in biologically relevant peptides. In this mini-review, we wish to provide a practical guideline of synthetic methods for the facile preparation of the building blocks required (chiral amino alkynes and azido acids) and their implementation in triazole-based peptide backbone engineering. While both 1,4and 1,5-disubstituted triazoles have been reported as amide-bond surrogates, e.g. in small non-peptide molecules, ${ }^{[8]}$ we focus here on 1,4-disubstituted 1,2,3-triazoles as trans-amide-bond surrogates in peptides. A comprehensive overview on the topic is beyond the scope of this mini-review and instead, we refer to some recent review articles covering various aspects of $\mathrm{CuAAC}$ and its applications, e.g. in peptide science. ${ }^{[9]}$

\section{Synthesis of Building Blocks}

Chiral $\alpha$-azido acid building blocks can be readily synthesized from the corresponding amino acids in a single step by a diazo transfer reaction with retention of the stereochemistry (Scheme 2). Two sulfonyl-based reagents are commonly employed for this transformation, triflyl azide (1) and, more recently, imidazolylsulfonyl azide (2) .

The synthesis of azides from amines with triflyl azide 1 was first described by Cavender and Shiner in 1972.[10] Reagent 1 enables the diazo transfer under mild conditions, compatible with common pro-

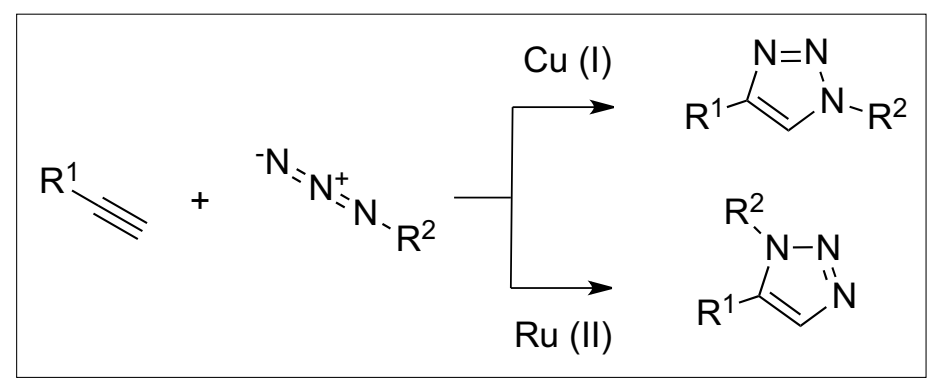

Scheme 1. Synthesis of 1,4- and 1,5-disubstituted 1,2,3-triazoles by CuAAc and RuAAC.

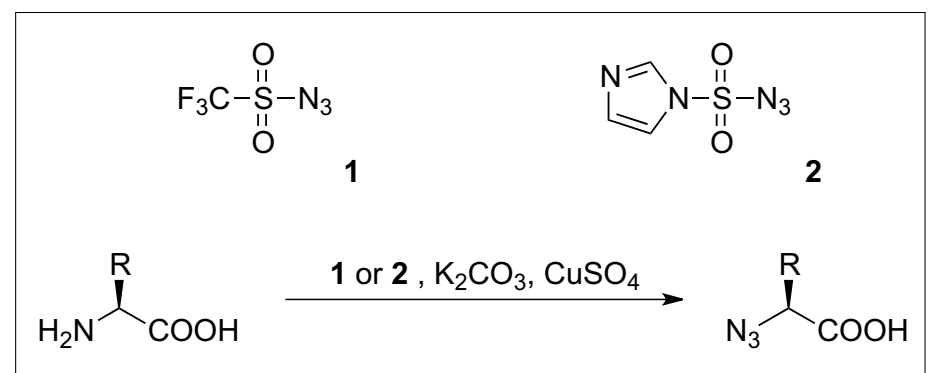

Scheme 2. Diazo transfer reactions of amino acids with triflyl azide 1 or imidazolylsulfonyl azide 2 yields chiral $\alpha$-azido acids.

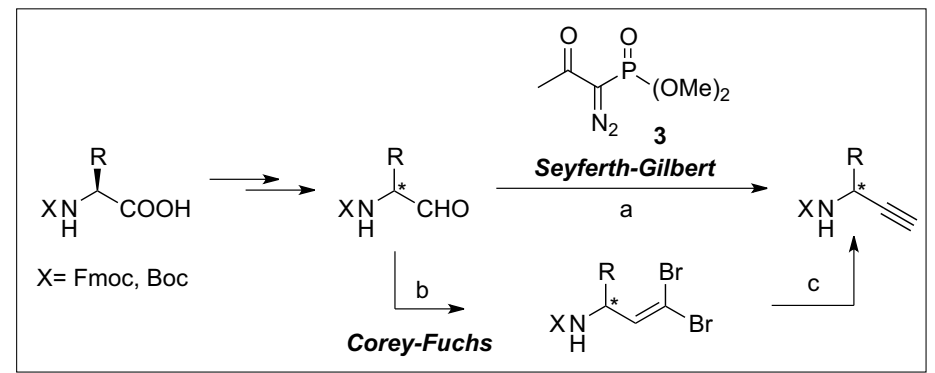

Scheme 3. Synthesis of $\alpha$-amino alkynes from $\alpha$-amino acids. a) $3, \mathrm{MeOH}, \mathrm{K}_{2} \mathrm{CO}_{3}$; b) $\mathrm{PPh}_{3}, \mathrm{CBr}_{4}$; c) $i$. n-BuLi, $-78^{\circ} \mathrm{C}$, ii. $\mathrm{MeOH}$.

tective groups of amino acid side chains. In 1996, Alper et al. improved the efficiency of the reaction by adding a divalent metal catalyst such as $\mathrm{Cu}$ (II) or $\mathrm{Zn}$ (II). ${ }^{[11]}$ However, the limited solubility of reagent $\mathbf{1}$ in polar solvents, usually required to dissolve $\alpha$-amino acids and metal catalysts, demands the careful optimization of the reaction conditions. ${ }^{[12]}$ In addition, triflyl azide 1 needs to be prepared freshly and is a potential explosive. The growing interest in amine-to-azide conversions has led to the development of another diazo transfer reagent based on an imidazolylsulfonyl moiety. Goddard-Borger and Stick proposed compound $\mathbf{2}$ as an inexpensive, safe, and easy to handle reagent for the synthesis of azides. ${ }^{[13]}$ With reagent $\mathbf{2}$, diazo transfer reactions can be performed safely on a gram-scale without the need of purification of the product by column chromatography. On the other hand, it has recently been reported that the hydrochloride of reagent $\mathbf{2}$ is highly hygroscopic and hydrolyzes slowly upon storage at room temperature. Thorough drying and storage at low temperature is recommended in order to prolong the chemical's shelf life. ${ }^{[14]}$ A direct comparison between reagents $\mathbf{1}$ and $\mathbf{2}$ suggests that the latter is equally efficient or superior to compound $\mathbf{1}$ in terms of yields and reaction times, but easier to handle.

In comparison to $\alpha$-azido acids, the preparation of chiral $\alpha$-amino alkynes from the corresponding $\alpha$-amino acids requires more synthetic steps and proceeds via an amino aldehyde intermediate, which is prone to racemization. The synthesis can be performed by either the Corey-Fuchs or Seyferth-Gilbert protocol (Scheme 3). ${ }^{[15]}$ The Corey-Fuchs homologation is a twostep procedure that requires a strong base (e.g. butyl lithium), inert conditions, and low temperature. ${ }^{[15 a]}$ The harsh basic conditions of this $\alpha$-amino acid homologation protocol bear the risk of racemization of the $\alpha$-amino aldehyde intermediate and thus, milder procedures would be preferable. ${ }^{[16]}$ In this context, the method reported by Bestmann and Ohira provides a solution..$^{[17]}$ The Bestmann-Ohira reagent $\mathbf{3}$ is commercially available, stable and requires neither a strong base, inert conditions, or low temperature. Dickson et al. have published a convenient one-pot procedure for the synthesis of terminal alkynes from methyl esters or Weinreb amides using reagent 3. ${ }^{[18]}$ The procedure described by the authors is particularly practical since it is scalable and avoids the isolation of the sensitive chiral $\alpha$-amino aldehyde intermediate. The homologation reaction described by Bestmann and Ohira is compatible with all amino acid side chains and classical protective groups used in solid phase peptide synthesis, and thus, represents 
the method of choice for the preparation of amino alkynes. Yet, verification of the optical purity of $\alpha$-amino alkyne products is recommended because partial racemization of certain amino acids in the course of the homologation reaction has recently been disputed in the literature. ${ }^{[16 a, 19]}$

\section{Synthesis of Triazolopeptides}

Azido- and alkyne building blocks can be built into the backbone of peptides by classical methods of solid-phase or solution peptide chemistry. ${ }^{[11 \mathrm{~b}, 20]}$ The triazole can be incorporated by different strategies. For example, the synthesis of small cyclic triazolo-peptides is usually achieved by solution-phase synthesis of the precursor followed by cyclisation employing the CuAAC (Scheme 4A; see examples in the following section). For the synthesis of longer triazolopeptides, the triazole can be incorporated during peptide elongation by coupling of a triazole-containing dipeptide mimic by classical SPPS methods (Scheme 4B). ${ }^{19 a]}$ Alternatively, the heterocycle can be installed on solid support by CuAAC of an alkyne precursor with an immobilized azide (Scheme 4C).[21] Finally, peptide fragments functionalized with azides and alkynes can be conjugated by CuAAC in solution, a strategy that has been proven useful as a ligation method for the assembly of larger proteins by CuAAC.[22]

Various experimentally determined reaction conditions have been reported for the CuAAC in solution and on solid support. While a thorough discussion of this aspect cannot be covered in this minireview, general practical aspects are discussed instead. The CuAAC is a metal-catalyzed, stepwise reaction that requires the presence of an active copper(I) species, an azide, an alkyne, and a proton acceptor.[6c] The reaction is very robust provided that all the aforementioned reactants are maintained in solution and that the copper(I) is not oxidized or disproportionated. In particular, the copper(I) source should be chosen in accordance to the solvent system employed. In general, copper(I) halides or $\left[\mathrm{Cu}\left(\mathrm{CH}_{3} \mathrm{CN}\right) \mathrm{PF}_{6}\right]$ are the preferred $\mathrm{Cu}(\mathrm{I})$ source to perform the CuAAC in organic solvents such as THF, DMSO, or toluene. Employment of these salts often requires an equivalent of an amine base such as triethylamine, diisopropylethylamine, or piperidine. ${ }^{[6 b, c]}$ For CuAAC conjugations in aqueous media, the in situ generation of $\mathrm{Cu}$ (I) catalyst from $\mathrm{Cu}$ (II) salts in the presence of a reducing agent, e.g. sodium ascorbate, has become the method of choice. Water-soluble sources of copper(I) have found frequent application because $\mathrm{Cu}$ (II) salts are often cheaper and available in higher purity than copper halides. The

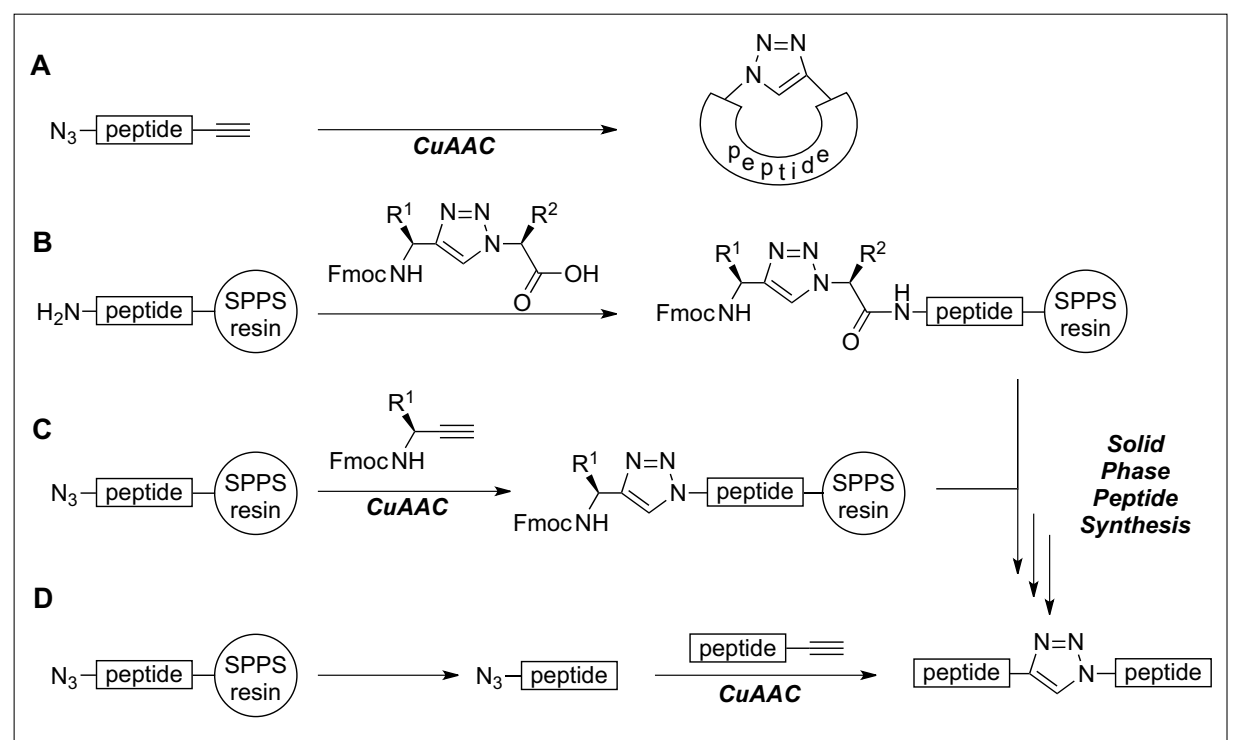

Scheme 4. Schematic representation of examples of the syntheses of triazolopeptides.

combination of $\mathrm{CuSO}_{4}$ and sodium ascorbate is particularly suited for CuAACs in polar solvents, such as water, alcohols, acetonitrile, DMF, or mixtures thereof.

If the substrates of the CuAAC possess potential coordination or chelation sites for copper species (e.g. carboxylates, phosphates, thiols, imidazoles, or amines) it is recommended to add a stabilizing ligand to prevent sequestering of the copper catalyst.[23] In addition, the presence of such ligands often accelerates the reaction and prevents the oxidation of the $\mathrm{Cu}(\mathrm{I})$ catalyst. ${ }^{[24]}$ A large number of stabilizing ligands for $\mathrm{Cu}(\mathrm{I})$ has been reported of which polytriazole-based systems have been employed most frequently. Among these readily accessible and structurally diverse ligands, ${ }^{[25]}$ tris-(benzyltriazolylmethyl)amine (TBTA) is commercially available and thus, most commonly used.

Even though some general procedures and reaction conditions can be defined for performing the $\mathrm{CuAAC}$ with peptidic substrates, it should be noted that individual optimizations may be required in certain cases in order to achieve optimal results.

\section{Examples of Biologically Active Triazolopeptides}

Because of its high efficiency and specificity, the CuAAC is particularly suited for macrocyclizations. As a consequence, the majority of triazole-containing peptidomimetics reported are cyclic peptides. One of the first examples published by Bock et al. describes the replacement of several amide bonds within the amino acid sequence of cyclic tyrosinase inhibitor 4 (Fig. 3). All the resulting triazolopeptides 5-7 were as active as the parent cyclopeptide $\mathbf{4}$ with regards to inhibition of the tyrosinase. [25] Interestingly, the use of $\mathrm{CuAAC}$ and thus the 1,2,3-triazole amide bond mimic, was required since the cyclization of compounds 4-7 could not be achieved by traditional peptide cyclization strategies due to the ring strain involved. ${ }^{[26,27]}$

Davis et al. have reported triazolopeptide 9, an analogue of sansalvamide A (8), an inhibitor of heat shock protein 90 with cytotoxic effect against several cancer cell lines. ${ }^{[28]}$ In their work, the authors studied the utility of different moieties as amidebond surrogates (1,2,3-triazole, oxazole,

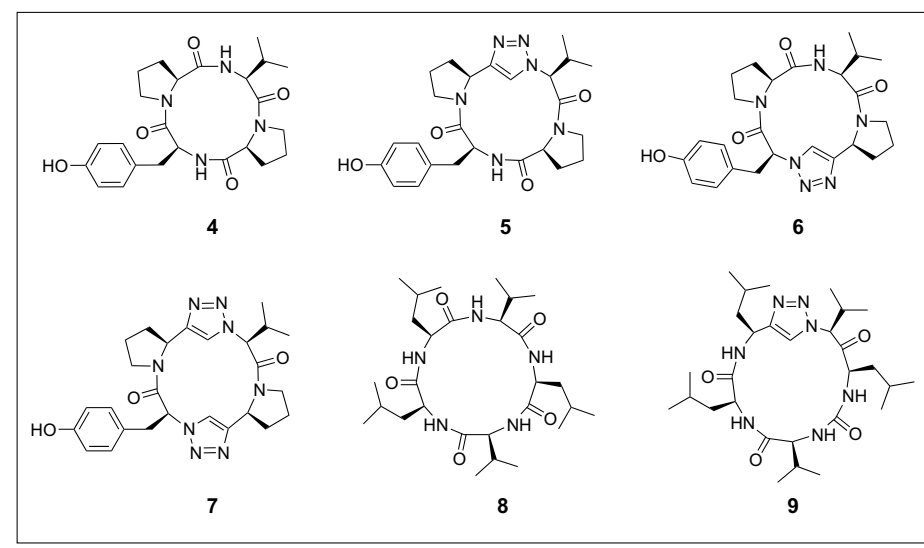

Fig. 3. Examples of cyclopeptides and their triazolo-analogues. 


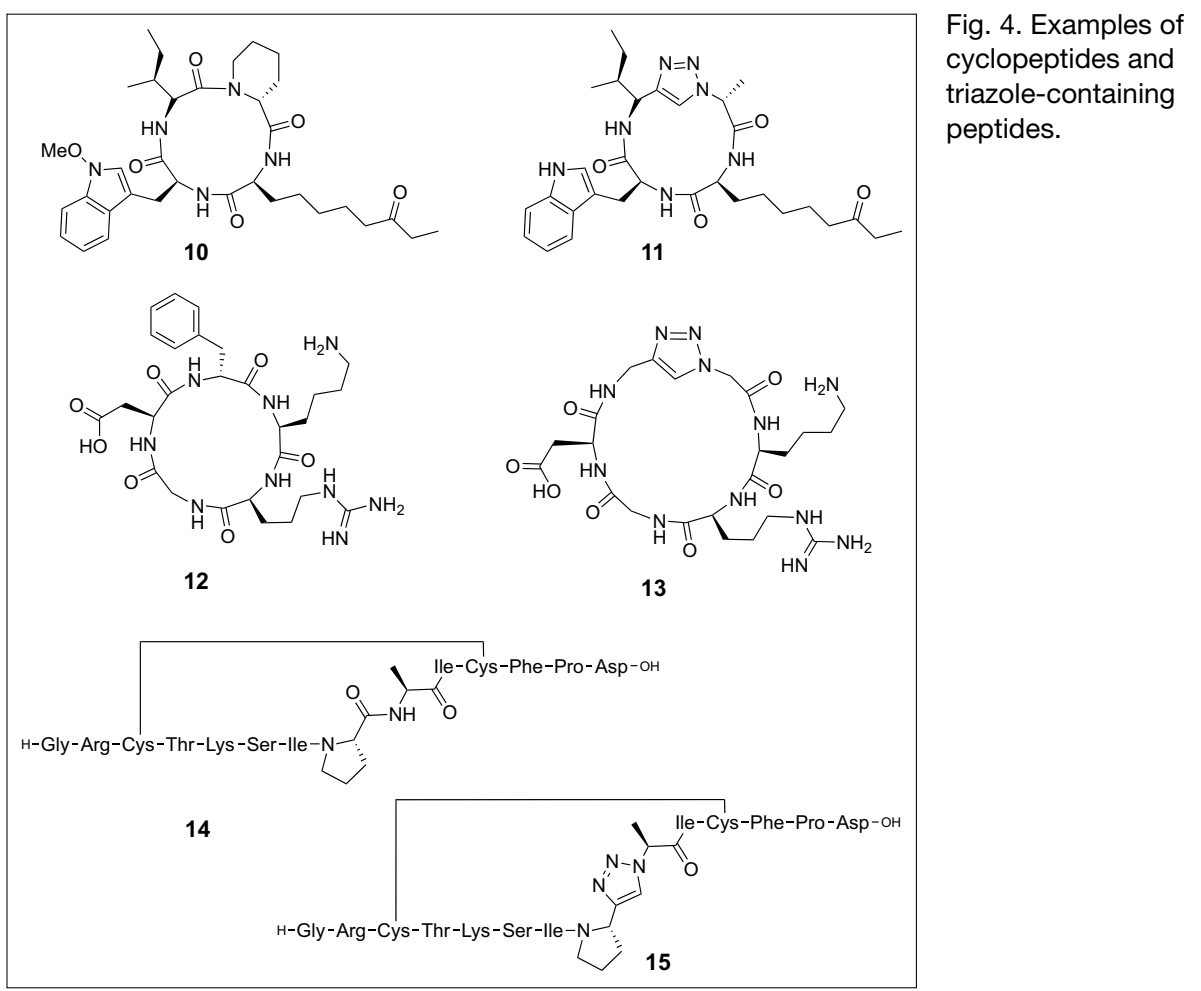

thiazole, or pseudoprolines) in the same position (Fig. 3). Evaluation of the compounds in vitro revealed that only triazole and thiazole heterocycles could be incorporated in the peptide without reduction of its cytotoxicity. In the case of triazole derivative 9 , the potency of reference compound $\mathbf{8}$ was maintained in terms of the inhibition of cell proliferation.

Horne et al. have replaced an isoleucyl-pipecolyl residue by an isoleucyl1,2,3-triazole-alanyl dipeptide mimic in apicidin 10, an inhibitor which binds with nanomolar affinity to several subtypes of histone deacetylases, (HDACs; Fig. 4). ${ }^{19 \mathrm{c}]}$ Interestingly, the modification changed the subtype-specificity of the original compound. Compared to apicidin 10, triazolopeptide 11 showed an 8-fold decreased inhibitory effect against HDAC-subtype 1 while the affinity against HDCA-subtype 3 was retained.

Cyclic peptides containing the amino acid sequence Arg-Gly-Asp (cRGD) bind to $\alpha_{\mathrm{v}} \beta_{3}$ integrins, which are involved in tumor angiogenesis and metastasis. Thus, a variety of cRGD-based compounds have been developed to target specifically tumor cells. In 2008, Liu et al. published the synthesis of a mimic of cyclopeptide $\mathbf{1 2}$ in which the D-phenylalanyl residue was replaced by a glycyl-1,2,3-triazole-glycyl dipeptide mimic (Fig. 4). ${ }^{[29]}$ In vitro studies performed with both compounds showed that triazolopeptide $\mathbf{1 3}$ had an affinity towards its receptor comparable to that of the parent compound $\mathbf{1 2}$.

Sunflower trypsin inhibitor 1 (SFTI-1) is a potent, cyclic protease inhibitor with a turn conformation crucial for its activity. Tischler et al. have replaced a transamide bond located between the prolyl and the alanyl residue of SFTI-1 analog 14 by a prolyl-1,2,3-triazole-alanyl dipeptoid (Fig. 4). ${ }^{[21]}$ In their work, the authors report the use of both 1,4- and 1,5-disubstituted 1,2,3-triazoles as amide-bond surrogates. As expected, only compound $\mathbf{1 5}$ derivatized with a 1,4-disubstituted triazole adopted a conformation close to the one of cyclopeptide $\mathbf{1 4}$ and exhibited nanomolar affinity against bovine trypsin.

The examples discussed above demonstrate the feasibility of the substitution of amide bonds by 1,2,3-triazole heterocycles in the backbone of peptides. Surprisingly, application of 1,2,3-triazoles as amidebond surrogates in linear, biologically active peptides has not yet been reported. Thus, we have recently started a research program directed towards the study of 1,2,3-triazoles as stabilizing elements in linear peptides. The results of these investigations will be reported in due time.

\section{Conclusions}

Despite the widespread use of the CuAAC in all fields of chemistry, relatively little attention has been paid so far to the potential of 1,4-disubstituted 1,2,3 triazoles as amide-bond bioisosteres for peptide backbone engineering. In this minireview, we provide a general and practical guideline for the synthesis of $\alpha$-azido acids and $\alpha$-amino alkynes and their use for amide-to-triazole substitution in the backbone of peptides with the intention to make this novel methodology for peptide modification more accessible. As more azide and alkyne building blocks become commercially available and the number of examples of reported triazolopeptides increases, we expect that the presented strategy will find broad application in the field of peptide science, $e$.g. for tumor targeting with peptides for drug delivery, imaging, or peptide receptor therapy.

\section{Acknowledgements}

We thank the Swiss National Science Foundation for financial support.

Received: February 27, 2013

[1] V. J. Hruby, Nat. Rev. Drug Discovery 2002, 1, 847.

[2] A. Choudhary, R. T. Raines, ChemBioChem 2011, 12, 1801

[3] N. A. Meanwell, J. Med. Chem. 2011, 54, 2529.

[4] E. Ko, J. Liu, L. M. Perez, G. Lu, A. Schaefer, K. Burgess, J. Am. Chem. Soc. 2010, 133, 462.

[5] a) A. Brik, J. Alexandratos, Y.-C. Lin, J. H. Elder, A. J. Olson, A. Wlodawer, D. S. Goodsell, C.-H. Wong, ChemBioChem 2005, 6, 1167; b) Y. R. Hua, A. H. Flood, Chem. Soc. Rev. 2010, $39,1262$.

[6] a) C. W. Tornøe, C. Christensen, M. Meldal, J. Org. Chem. 2002, 67, 3057; b) V. V. Rostovtsev, L. G. Green, V. V. Fokin, K. B. Sharpless, Angew. Chem., Int. Ed. 2002, 41, 2596; c) M. Meldal, C. W. Tornøe, Chem. Rev. 2008, 108, 2952.

[7] L. Zhang, X. Chen, P. Xue, H. H. Y. Sun, I. D. Williams, K. B. Sharpless, V. V. Fokin, G. Jia, $J$. Am. Chem. Soc. 2005, 127, 15998.

[8] a) G. Appendino, S. Bacchiega, A. Minassi, M. G. Cascio, L. De Petrocellis, V. Di Marzo, Angew. Chem. Int. Ed. 2007, 46, 9312; b) T. Lee, M. Cho, S.-Y. Ko, H.-J. Youn, D. J. Baek, W.-J. Cho, C.-Y. Kang, S. Kim, J. Med. Chem. 2007, 50,585

[9] D. S. Pedersen, A. Abell, Eur. J. Org. Chem. 2011, 2399.

[10] C. J. Cavender, V. J. Shiner, J. Org. Chem. 1972, 37, 3567.

[11] a) P. B. Alper, S.-C. Hung, C.-H. Wong, Tetrahedron Lett. 1996, 37, 6029; b) J. T. Lundquist, J. C. Pelletier, Org. Lett. 2001, 3, 781.

[12] P. T. Nyffeler, C.-H. Liang, K. M. Koeller, C.-H. Wong, J. Am. Chem. Soc. 2002, 124, 10773.

[13] E. D. Goddard-Borger, R. V. Stick, Org. Lett. 2007, 9, 3797

[14] a) E. D. Goddard-Borger, R. V. Stick, Org. Lett. 2011, 13, 2514; b) N. Fischer, E. D. GoddardBorger, R. Greiner, T. M. Klapotke, B. W. Skelton, J. Stierstorfer, J. Org. Chem. 2012, 77, 1760.

[15] a) E. J. Corey, P. L. Fuchs, Tetrahedron Lett. 1972, 3769; b) D. Seyferth, R. S. Marmor, P. Hilbert, J. Org. Chem. 1971, 36, 1379; c) J. C. Gilbert, U. Weerasooriya, J. Org. Chem. 1979, 44, 4997.

[16] a) G. Reginato, A. Mordini, F. Messina, A Degl'Innocenti, G. Poli, Tetrahedron 1996, 52, 10985; b) J. Y. L. Chung, J. T. Wasicak, Tetrahedron Lett. 1990, 31, 3957.

[17] a) S. Müller, B. Liepold, G. J. Roth, H. J. Bestmann, Synlett 1996, 521; b) S. Ohira, Synth. Commun. 1989, 19, 561.

[18] H. D. Dickson, S. C. Smith, K. W. Hinkle, Tetrahedron Lett. 2004, 45, 5597.

[19] a) A. Tam, U. Arnold, M. B. Soellner, R. T. 
Raines, J. Am. Chem. Soc. 2007, 129, 12670; b) E. Ko, K. Burgess, Org. Lett. 2011, 13, 980; c) S. W. Horne, C. A. Olsen, J. M. Beierle, A. Montero, R. M. Ghadiri, Angew. Chem. Int. Ed. 2009, $48,4718$.

[20] J. Alsina, F. Albericio, Pept. Sci. 2003, 71, 454.

[21] M. Tischler, D. Nasu, M. Empting, S. Schmelz, D. W. Heinz, P. Rottmann, H. Kolmar, G. Buntkowsky, D. Tietze, O. Avrutina, Angew. Chem. Int. Ed. 2012, 51, 3708.
[22] I. E. Valverde, F. Lecaille, G. Lalmanach, V. Aucagne, A. F. Delmas, Angew. Chem. Int. Ed. 2012, 51, 718 .

[23] V. Hong, S. I. Presolski, C. Ma, M. G. Finn, Angew. Chem. Int. Ed. 2009, 48, 9879.

[24] a) S. I. Presolski, V. Hong, S.-H. Cho, M. G. Finn, J. Am. Chem. Soc. 2010, 132, 14570; b) V. O. Rodionov, S. I. Presolski, D. Diaz Diaz, V. V. Fokin, M. G. Finn, J. Am. Chem. Soc. 2007, 129, 12705.
[25] a) T. R. Chan, R. Hilgraf, K. B. Sharpless, V. V. Fokin, Org. Lett. 2004, 6, 2853; b) Z. Zhou, C. J. Fahrni, J. Am. Chem. Soc. 2004, 126, 8862.

[26] V. D. Bock, D. Speijer, H. Hiemstra, J. H. v. Maarseveen, Org. Biomol. Chem. 2007, 5, 971.

[27] U. Schmidt, J. Langner, J. Pept. Res. 1997, 49,

[28] M. R. Davis, E. K. Singh, H. Wahyudi, L. D. Alexander, J. B. Kunicki, L. A. Nazarova, K. A. Fairweather, A. M. Giltrap, K. A. Jolliffe, S. R. McAlpine, Tetrahedron 2012, 68, 1029.

[29] Y. Q. Liu, L. H. Zhang, J. P. Wan, Y. S. Li, Y. H. Xu, Y. J. Pan, Tetrahedron 2008, 64, 10728. 